\title{
Application of OSL dosimetry and 3D printed phantom for comparison of calculation algorithms for VMAT treatment planning
}

\author{
Daniel Villania (dvillani@ipen.br); Carolina dos Santos Moreno ${ }^{b}$ \\ (carolina.moreno@einstein.br); Roberto Kenji Sakurabab \\ (roberto.sakuraba@einstein.br) and Letícia Lucente Camposa (Icrodri@ipen.br)
}

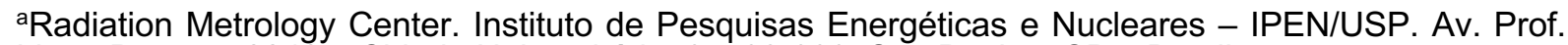
Lineu Prestes, 2242 - Cidade Universitária, 05508-000, São Paulo - SP - Brazil.

${ }^{\mathrm{b}}$ Radiation Therapy Department. Hospital Israelita Albert Einstein. Av. Albert Einstein, 627 - Morumbi, 05652-900, São Paulo - SP, Brazil.

\section{DOI: https://doi.org/10.34019/2674-9688.2019.v1.28227}

\begin{abstract}
The application of new commercial and industrial technologies in the fields of dosimetry and medical physics is of great interest to the scientific community, both to validate existing protocols and to develop new methodologies. The popularization of 3D printing techniques has been analyzed as a great advantage in quality control in complex treatment techniques, such as radiotherapy and the development of patient simulators. Portable dosimetry systems such as Landauer MicroStar OSL system are versatile and their use in quality control is of great importance. The aim of this paper is to compare two of the most used dose calculation algorithms used in Varian Eclipse TPS - AAA and Acuros XB - for treatment planning of multiple brain metastases using a 3D printed anthropomorphic phantom and the OSL InLight system for experimental dosimetry validation. A 3D printed anthropomorphic skull phantom was submitted to a CT scan and planed five target volumes. In order of comparison, two dose calculations were performed in the Varian Eclipse 13.6 TPS with "Alabama technique", using the Varian's AAA and AXB algorithms, and treatment delivered with $6 \mathrm{MV}$ photon beam of a Varian TrueBeam linear accelerator. Landauer nanoDot dosimeters were positioned inside each of the five target volumes planned and the experimental dosimetric results were compared with the algorithms' calculated doses. The findings of this work indicate that ACUROS XB calculates more accurate doses compared with AAA, with all the experimental agreements better than $96.0 \%$, probably because of the heterogeneity corrections. The uncertainty analysis of the InLight system device is enough to sustain the dosimetric uncertainties below $3.0 \%$, validating the results.
\end{abstract}

Keywords: 3D printed phantom; Alabama Technique; OSL Dosimetry; Varian AAA; Varian Acuros XB 


\section{Introduction}

Metastatic brain carcinoma affects $20 \%$ to $30 \%$ of patients with systemic cancer and is recognized as the most common intracranial tumor in adults. Brain metastases are also the most frequent metastatic neurological complication of systemic cancer and are second only to metabolic encephalopathies as a case of central nervous system dysfunction in cancer patients. From 20 to 40 percent of lung cancer patients may also develop brain metastases [1-3].

Single brain metastasis refers to an apparent single cerebral lesion, with no consideration over the extent of cancer elsewhere in the body. The definition of 'solitary brain metastasis' is used to describe the relatively rare occurrence of a single brain metastasis, occurring in only one-third to one-fourth of patients with cerebral metastases [1].

Treatments are a combination of whole brain radiotherapy (WBRT), stereotactic radiosurgery (SRS), surgery, and chemotherapy. SRS provides effective local control especially for deep-seated small metastases [4]. With widespread use and improved quality of magnetic resonance (MR) imaging, more patients with brain metastases are detected at early stage [1].

The Volumetric modulated arc therapy (VMAT; RapidArc, Varian Medical systems, Palo Alto, CA) has already become a widely used plan optimization and treatment delivery platform in which the Multileaf Collimator (MLC) dynamically conforms the target while the gantry rotates, and radiation can be delivered efficiently and accurately in one or more dynamically modulated arcs [5].

Typically, when treating multiple brain metastases, linear accelerator-based VMAT SRS techniques have used a set-up with multi-isocenter, in which each lesion is targeted and treated sequentially. This type of treatment has duration time proportional to the number of lesions in the patient, and can vary from 20 minutes up to one hour. In order to reduce treatment time per section, combining good dose compliance, low toxicity and good treatment quality, Clark, et al. (2010) [6] studied the feasibility of single-isocenter VMAT techniques to treat multiple brain lesions, reporting later [7] a more detailed outcome, applied in their institution. In this called "Alabama" technique, a single-isocenter/multi-arc cranial radiosurgery approach is used with VMAT (RapidArc), and the number of arcs depends on patients need.

In their study, the median number of arcs utilized was 2, usually consisting of an axial arc spanning 358 degrees with couch angle of 0 degrees; combined with a vertex arc (couch angle 90 degrees) spanning 171 degrees. Another common multi-arc arrangement included 4-arc plans, consisting of a 358-degree axial arc (couch angle 0 degrees) and 3 additional arcs spanning 171 degrees, utilizing the couch angles of 45,90 , and 315 degrees to create a non-coplanar arc fields array. The single arc arrangements utilized a 0-degree couch angle 358-degree arc was more likely to be utilized in single target cases [7].

The Radiation Therapy Center of the Hospital Israelita Albert Einstein (HIAE) uses the Alabama technique approach in the treatment of their patients. For the VMAT treatments it is used the Varian Eclipse 13.6 treatment planning system (TPS) with the Analytical Anisotropic Algorithm (AAA) and Acuros XB Advanced Dose Calculation Algorithm (AXB) available. The AAA uses convolution-superposition methods to calculate the dose [8] and the AXB uses the fundamental radiation transport theory, based on the grid-based Boltzmann solver (GBBS) 
[9]. Many studies have been published to compare these different algorithms with Monte Carlo (MC) simulations and/or validate with dosimetric data [9-16].

The Optically stimulated luminescence OSL dosimetry has become one of most used techniques for radiation dosimetry nowadays, especially after the improvement of Landauer's Luxel $^{\mathrm{TM}}$ and creation of Inlight ${ }^{\mathrm{TM}}$ System. The Inlight ${ }^{\mathrm{TM}}$ System is a simple and efficient commercial system for use in OSL dosimetry that recently has been used as tool to help validating radiation therapy dosimetry [17-20].

The application of new commercial and industrial technologies in the fields of dosimetry and medical physics is of great interest to the scientific community, both to validate existing protocols and to develop new methodologies. The popularization of 3D printing techniques has been analyzed as a great advantage in quality control in complex treatment techniques, such as radiotherapy and the development of patient simulators. In order to compare the performance of the Varian $\mathrm{AAA}$ and Acuros $\mathrm{XB}$ dose calculation algorithms in the treatment planning of multiple brain metastases using the 'Alabama' methodology, this work reports a comparative study between dose calculation algorithms and experimental data obtained with the InLight OSL dosimetry system and a 3D printed anthropomorphic phantom.

\section{Material and Methods}

To this work it was used a head 3D printed tissue-equivalent anthropomorphic phantom and the commercial OSL system inLight microStar and nanoDot dosimeters in order to determine experimentally the accuracy of two of the most used calculation algorithms in radiation therapy - Varian $A A A$ and Varian Acurus $X B(A X B)$ - for treatment planning of multiple brain metastasis. The OSL system was commissioned to obtain the experimental absorbed doses of five targeted tumors inside the 3D printed phantom. Using the linear accelerator Varian TrueBeam, the planned treatment using "Alabama technique" was delivered to the phantom and the experimental absorbed doses compared with AAA and AXB calculated doses.

\section{Results and Discussion}

\subsection{D printed anthropomorphic phantom}

In order to simulate a patient with metastatic brain carcinoma an anthropomorphic skull phantom printed in $3 \mathrm{D}$ was used. This phantom is tissue equivalent developed from a computerized tomography of a patient and printed in 3D by BioArchitects. This way, it was possible to simulate a real treatment, with reliability in the structures anatomy and media heterogeneity. The 3D printed anthropomorphic phantom is showed in Figure. 1. 

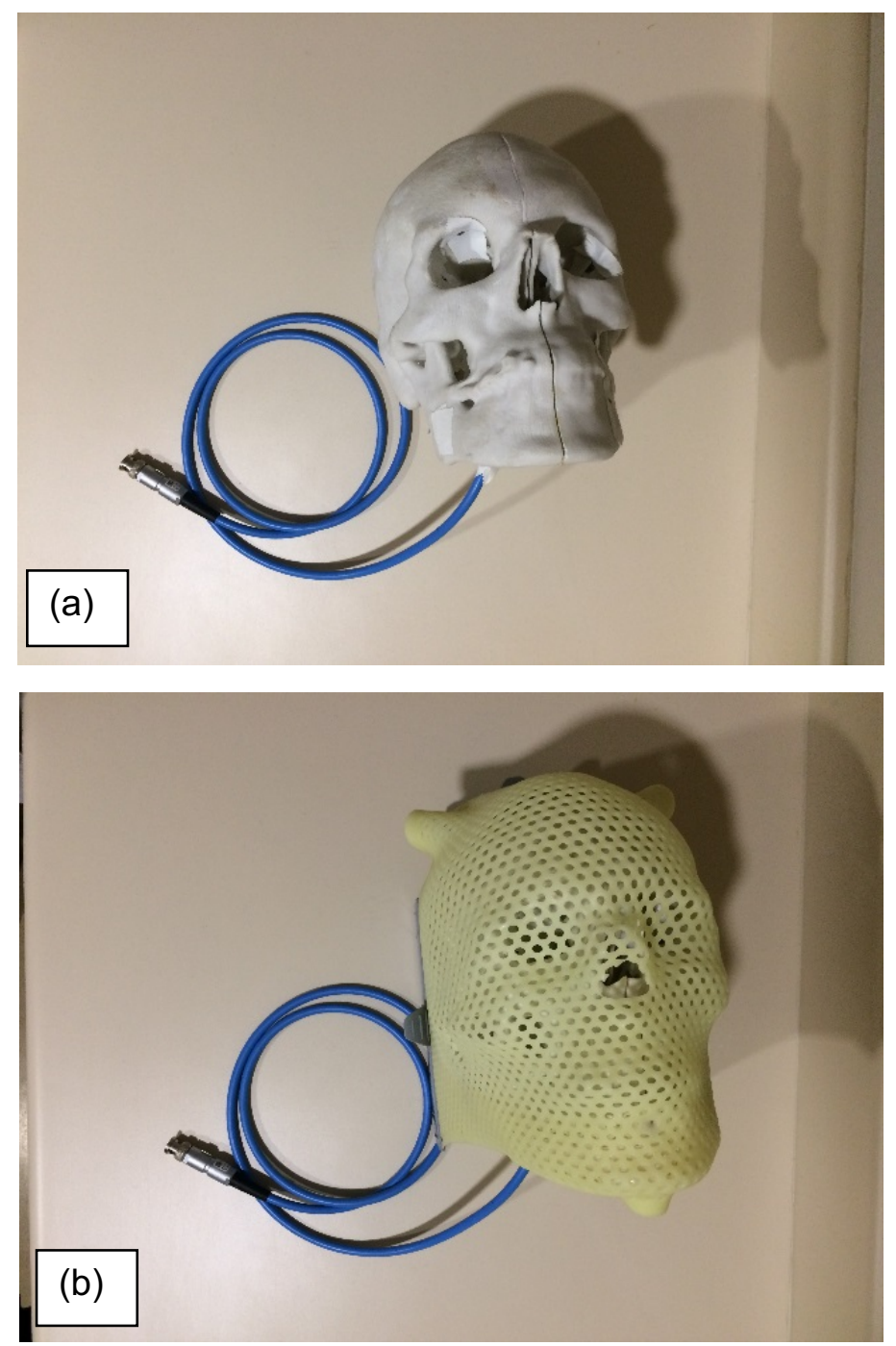

Figure 1. 3D printed anthropomorphic phantom used in this study. (a) Frontal view of the phantom. (b) Phantom with respective thermoplastic mask for couch positioning.

The phantom was submitted to a CT scan (GE tomography, model CT LightSpeed RT 16), and five target volumes to be treated were planned surrounding the brain region with the 4-arc 'Alabama' technique and OSL dosimetry measurements compared with AAA and Acuros XB calculated doses.

\subsection{VMAT treatment and dose calculations}

The plan was created using the 6 MV SRS photon beam, Flatness Filter Free (FFF) and maximal dose rate of $1400 \mathrm{MU} / \mathrm{min}$. A 4-arc plan of the "Alabama" technique was created in the Varian Eclipse 13.6 TPS with grid line of $1.0 \mathrm{~mm}$. In order compare the calculated doses, to the same plan we used both $A A A$ and $A X B$ algorithms to perform the dose calculations of each target volume planed. The dose-distribution achieved with the 4-arc plan treatment with 'Alabama' technique can be observed in Figure 2 (a), and de visual distribution of the arcs in Figure 2 (b). To this treatment plan, two dose calculations were performed with AAA and AXB algorithms. 

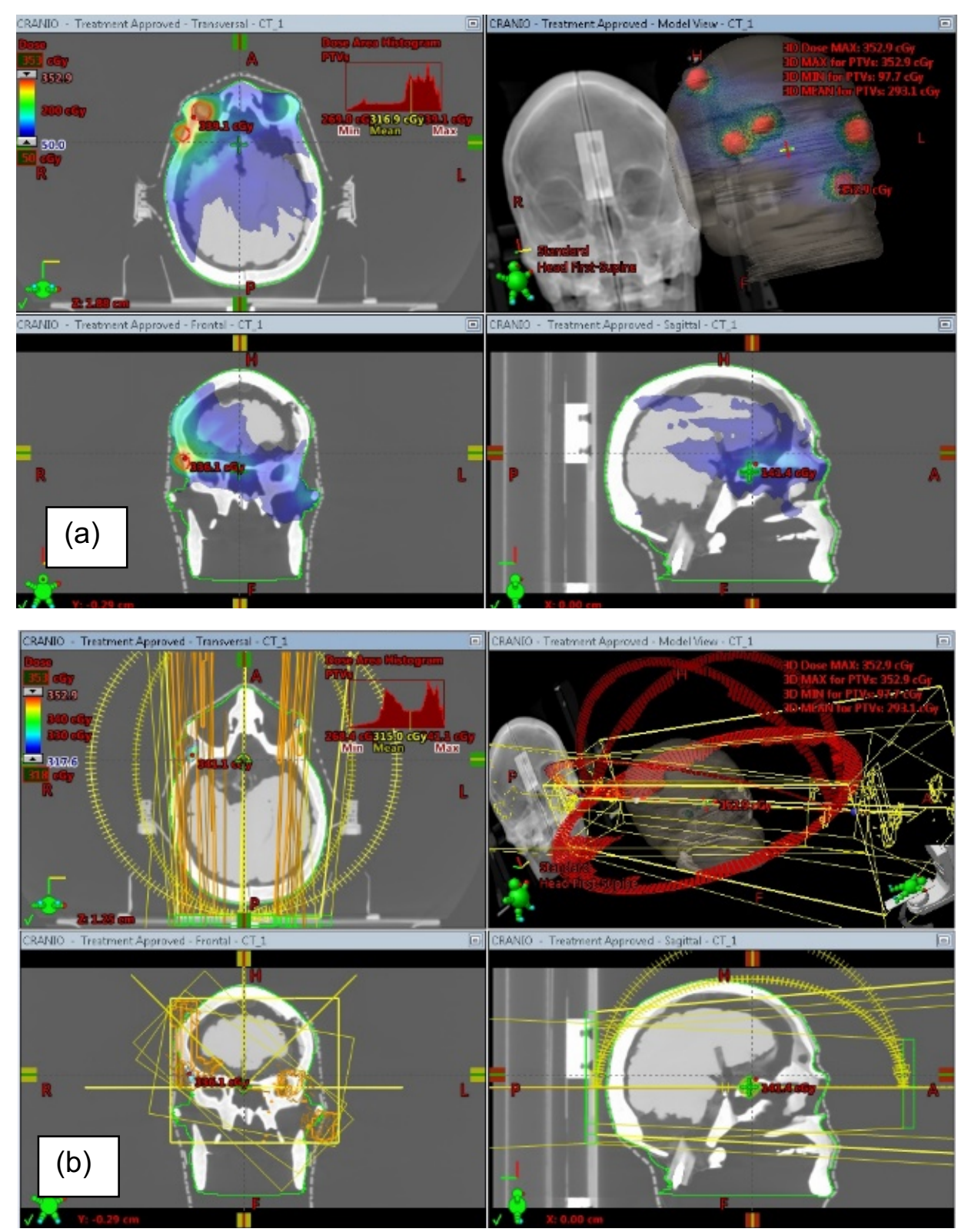

Figure 2. VMAT planned treatment. (a) dose distribution within the 5 targets and (b) 4-Arc plan distribution using "Alabama Technique".

The clinical irradiations were carried out on the Varian TrueBeam ${ }^{\mathrm{TM}}$ (Varian Medical Systems, Inc., Palo Alto, California) linear accelerator of the Radiotherapy Center of the Hospital Israelita Albert Einstein (HIAE), equipped with the Varian High Definition (HD) 120 MLC. It has 120 has 60 leaf pairs with a total field length of $22 \mathrm{~cm}$, with leaf widths of $2.5 \mathrm{~mm}$ in the central, $8 \mathrm{~cm}$ and $5 \mathrm{~mm}$ in the outer portion of the field. The leaf transmission of this $\mathrm{MLC}$ is $1 \%$ for $6 \mathrm{MV}$.

The phantom was positioned using image guidance of cone-beam CT, and positioning corrections needed were performed with the robotic. Landauer nanoDot dosimeters were positioned inside each of the five target volumes planned and the irradiation set-up is showed in Figure 3. 


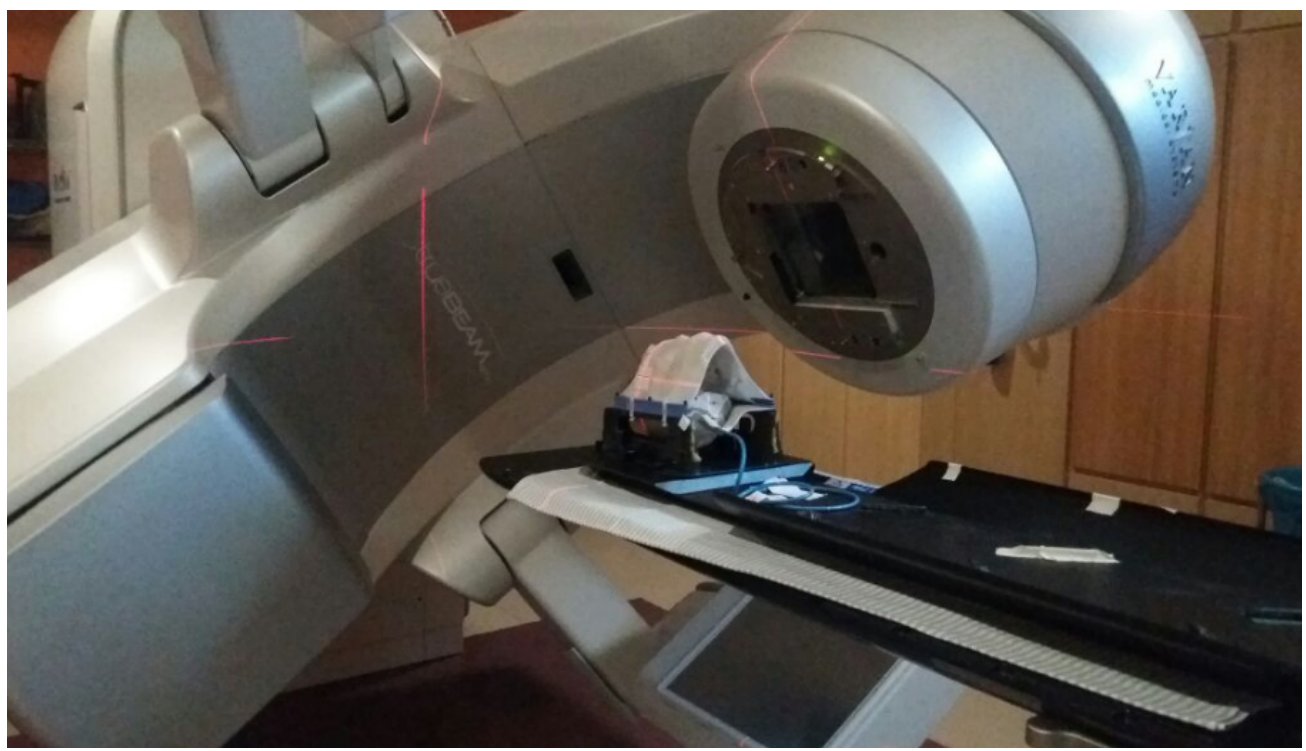

Figure 3. Irradiation set-up. Anthropomorphic 3D printed phantom positioned over the robotic couch of the Varian TrueBeam of the HIAE

\subsection{Experimental dosimetric evaluation}

The experimental measurements to be compared with the calculated doses were obtained with OSL dosimetry. The material used in this study was the well-known $\mathrm{Al}_{2} \mathrm{O}_{3}: \mathrm{C}$ nanoDot optically stimulated dosimeters (OSLDs) manufactured by Landauer Inc. (Landauer Inc., Glenwood, IL). They are $5 \mathrm{~mm}$ diameter, $0.2 \mathrm{~mm}$ thick disk-shaped $\mathrm{Al}_{2} \mathrm{O}_{3}: \mathrm{C}$, encased in a light tight black plastic with dimensions of $10 \times 10 \times 2 \mathrm{~mm}^{3}$ (Figure $4 \mathrm{~b}$ ). The samples were granted from SAPRA Landauer Serviços de Acessoria e Proteção Radiológica Ltda.
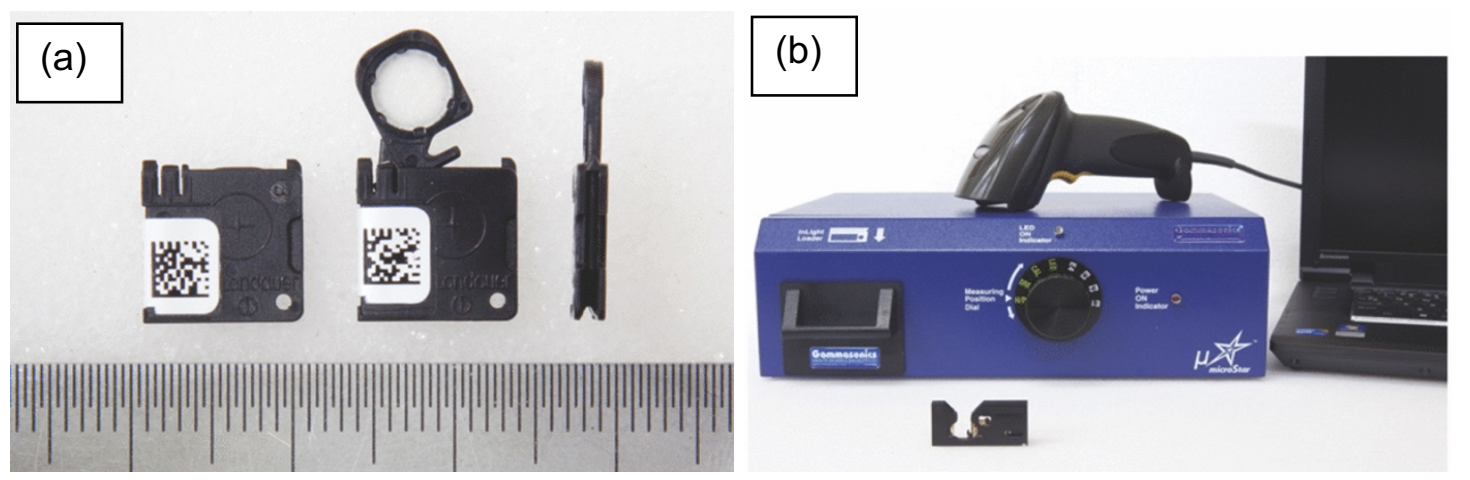

Figure 4. Commercial OSL system used in this work. (a) NanoDot OLDs and (b) InLight MicroStar reader.

The nanoDots readout were performed using the InLight System from HIAE. It consists of a microStar reader connected to a notebook and signal-to-dose calculations performed by $\mu$ Star software. The unit uses Light Emitting Diodes (LED) emitting green stimulation light (wavelength of $532 \mathrm{~nm}$ ) [21]. Optical beaching of the OSL samples was performed using an Ourolux ${ }^{\circledR} 1.3 \mathrm{~W}$ of power lamp, composed of 30 blue LEDs. The sensitive $\mathrm{Al}_{2} \mathrm{O}_{3}: \mathrm{C}$ disks were exposed to the light for $\sim 12 \mathrm{~h}$ prior every further irradiation and the 'background' signal was 
read for each dosimeter before new uses. Dosimetric characterization of the OSL system was performed following the recommended guidelines [18, 22] for $6 \mathrm{MV}$ photon beam using the Varian TrueBeam linear accelerator. The total uncertainty of the OSL reading device was evaluated by means of $C V_{Q, p}$, calculated using Equation 1

$$
C V_{\% 6}=\left(\frac{\sigma_{R_{Q A}}}{\overline{R_{Q A}}}\right) \cdot 100
$$

where $\overline{R_{Q A}}$ is the mean of $10 \mathrm{OSL}$ repeated readouts of a QA dosimeter, and $\sigma_{R_{Q A}}$ the standard deviation of the 10 readouts. This test results in 1- $\sigma$ confidence interval of the unit and is extremely important for detecting any problems related to the reader's mechanics and/or poor repeatability of the QA nanoDot. For radiation therapy measurements, this should be less than $2.0 \%$ [21].

The presented OSL experimental results of the absorbed doses are the average of five dosimeters readout, and the error bars are the standard deviation the mean. The calculations of the absorbed doses and the OSL reader tests were performed with $\mu$ Star software, and any additional evaluation carried out with Microsoft Excel 2016 software. Graphics were plotted using OriginPro $8.1^{\mathrm{TM}}$, and, the all the absorbed doses were expressed in "cGy", due to clinical applications and regarding a better understanding for Medical Physics field.

In this study, the OSL reading device remained stable during all measurements, with dosimetric characterization performed according to literature and with $C V_{\text {甲 }} \leq 1.4 \%$. Table 1 show the comparison between the algorithms, the doses given by the two algorithms calculated and experimental doses obtained with OSL dosimetry. Target identifications are shown in Figure 5 (a) and Figure 5 (b) shows the agreement between all the obtained data.

Table 1. Calculated doses using $A A A$ and ACUROS XB algorithms and experimental OSLD measurements

\begin{tabular}{|c|c|c|c|c|c|c|c|c|c|}
\hline & \multicolumn{6}{|c|}{ Calculation Algorithms } & \multirow{2}{*}{\multicolumn{3}{|c|}{$\begin{array}{c}\text { Experimental } \\
\text { OSLDs }\end{array}$}} \\
\hline & \multicolumn{3}{|c|}{ AAA } & \multicolumn{3}{|c|}{ ACUROS XB } & & & \\
\hline Structure & $\begin{array}{c}\text { Min } \\
\text { Dose } \\
\text { (cGy) }\end{array}$ & $\begin{array}{c}\text { Max } \\
\text { Dose } \\
\text { (cGy) }\end{array}$ & $\begin{array}{c}\text { Mean Dose } \\
\text { (cGy) }\end{array}$ & $\begin{array}{c}\text { Min } \\
\text { Dose } \\
\text { (cGy) }\end{array}$ & $\begin{array}{c}\text { Max } \\
\text { Dose } \\
\text { (cGy) }\end{array}$ & $\begin{array}{c}\text { Mean Dose } \\
\text { (cGy) }\end{array}$ & $\begin{array}{c}\text { Min } \\
\text { Dose } \\
\text { (cGy) }\end{array}$ & $\begin{array}{l}\text { Max } \\
\text { Dose } \\
\text { (cGy) }\end{array}$ & $\begin{array}{c}\text { Mean Dose } \\
\text { (cGy) }\end{array}$ \\
\hline 1 & 148.3 & 309.6 & $241.9 \pm 34.9$ & 207.0 & 317.9 & $277.8 \pm 23.4$ & 261.6 & 294.5 & $270.2 \pm 5.7$ \\
\hline 2 & 300.1 & 333.2 & $318.2 \pm 7.7$ & 301.6 & 354.7 & $333.7 \pm 8.6$ & 306.6 & 363.4 & $330.8 \pm 9.2$ \\
\hline 3 & 316.3 & 333.4 & $327.0 \pm 4.0$ & 328.8 & 353.4 & $345.0 \pm 4.8$ & 326.2 & 360.1 & $345.7 \pm 7.6$ \\
\hline 4 & 287.8 & 334,0 & $310.9 \pm 9.9$ & 320,0 & 350,0 & $338.0 \pm 5.7$ & 332.8 & 361.2 & $352.3 \pm 5.6$ \\
\hline 5 & 265.0 & 332.7 & $312.9 \pm 9.8$ & 300.9 & 535.5 & $337.1 \pm 8.6$ & 305.2 & 372.6 & $341.2 \pm 15.1$ \\
\hline
\end{tabular}



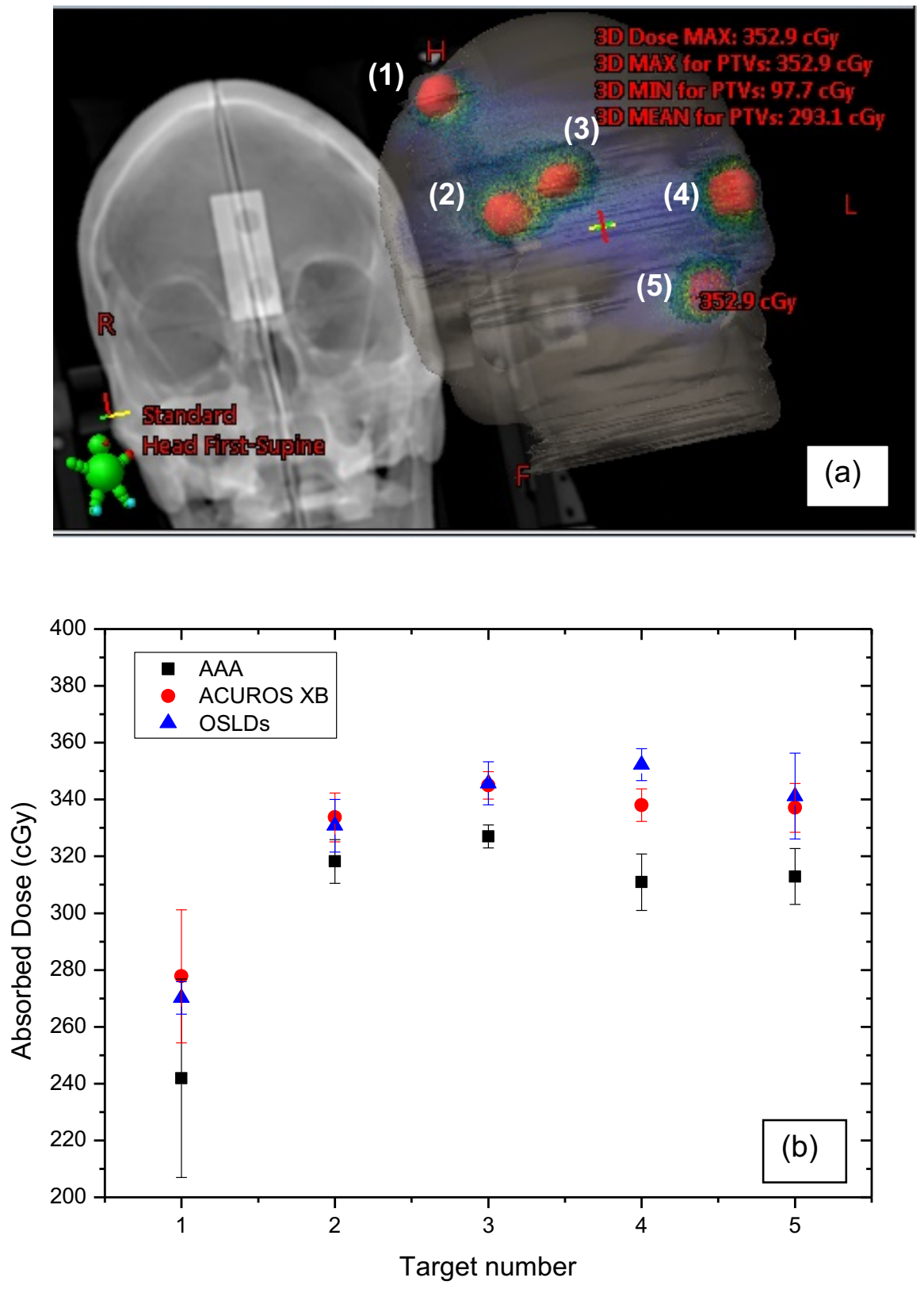

Figure 5. (a) Target identification. (b) Agreement between the mean absorbed doses obtained with the experimental OSL data in comparison with AAA and ACUROS XB calculations.

Through the analysis of the presented results one can observe that the experimental measurements using the OSLDs show agreement of $97.3 \%, 99.1 \%, 99.9 \%, 96.0 \%$ and $98.8 \%$ for the targets 1 to 5 respectively for the ACUROS XB algorithm calculated doses.

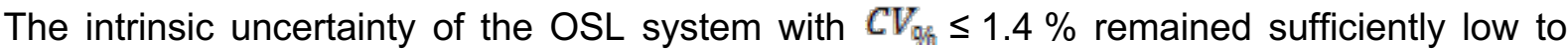
validate the experimental data obtained. Han et al. (2012) [14] also found better agreement of $A X B$ with their experimental TLD and film measurements. In our treatment simulation, AAA underestimates the doses in every target studied, unlike the clinical study performed by Kan et al. (2012) in which observed an overestimation of up to $10 \%$ in the nasopharyngeal 
region.

\section{Conclusions}

The experimental findings of this work indicate that ACUROS XB calculation algorithm results more accurate doses compared with AAA, with all the experimental agreements better than $96.0 \%$. This can be explained by the improved heterogeneity corrections on this version of the algorithm. The intrinsic precision and uncertainty of the InLight system device is enough to sustain the dosimetric uncertainties below $3.0 \%$, validating the results.

\section{Acknowledgments}

The authors would like to thank CNPq, CNEN and FAPESP for the financial support, SAPRA Landauer for the samples and technical support and to the radiation therapy staff of the Hospital Israelita Albert Einstein for the irradiations.

\section{References and Notes}

[1] Patchell, R. A. Brain metastases. Neurol Clin 1991;9:817-24.

[2] Nussbaum, E. S. et al. Brain metastases. Histology, multiplicity, surgery, and survival. Cancer 1996;78:1781-8.

[3] Stewart BW, Wild C: World Cancer Report 2014. 2014 International Agency for Research on Cancer, et al Lyon, France International Agency for Research on Cancer

[4] Smith ML, Lee JY. Stereotactic radiosurgery in the management of brain metastasis. Neurosurg Focus 2007;22:E5.

[5] Otto, K., 2008. Volumetric modulated arc therapy: IMRT in a single gantry arc. Med. Phys. 35 (1), 310-317.

[6] Clark, G. M., et al. Feasibility of single isocenter volumetric modulated arc radiosurgery for treatment of multiple brain metastases. Int $J$ Radiat Oncol Biol Phys. 2010;76:296-302.

[7] Clark, G. M., et al. "Plan quality and treatment planning technique for single isocenter cranial radiosurgery with volumetric modulated arc therapy." Practical radiation oncology 2.4 (2012): 306-313.

[8] Fogliata, A. et al. "Dosimetric validation of the anisotropic analytical algorithm for photon dose calculation: fundamental characterization in water." Physics in medicine and biology 51.6 (2006): 1421.

[9] Fogliata, A. et al. "Dosimetric validation of the Acuros XB Advanced Dose Calculation algorithm: fundamental characterization in water." Physics in medicine and biology 56.6 (2011): 1879.

[10] Fogliata, A. et al. "Dosimetric evaluation of Acuros XB Advanced Dose Calculation algorithm in heterogeneous media." Radiation oncology 6.1 (2011): 82.

[11] Van Esch, Ann, et al. "Testing of the analytical anisotropic algorithm for photon dose calculation." Medical physics 33.11 (2006): 4130-4148.

[12] Vassiliev, O.N., Wareing TA, McGhee J, Failla G, Salehpour MR, Mourtada F.

Validation of a new grid based Blotzmann equation solver for dose calculation in radiotherapy with photon beams. Phys Med Biol. 2010;55:581-98.

[13] daRosa, L. A. R., et al. "Percentage depth dose evaluation in heterogeneous media using thermoluminescent dosimetry." Journal of Applied Clinical Medical Physics 11.1 (2010): 117-127. 
[14] Han, T. et al. "Experimental validation of deterministic Acuros XB algorithm for IMRT and VMAT dose calculations with the Radiological Physics Center's head and neck phantom." Medical physics 39.4 (2012): 2193-2202.

[15] Kan, M. W, Leung, L. H, Yu, P. K. Verification and dosimetric impact of Acuros $\mathrm{XB}$ algorithm on intensity modulated stereotactic radiotherapy for locally persistent nasopharyngeal carcinoma. Med Phys. 2012;39:4705-14. [PubMed: 22894395]

[16] Rana, S. and Rogers, K. "Dosimetric evaluation of Acuros XB dose calculation algorithm with measurements in predicting doses beyond different air gap thickness for smaller and larger field sizes." Journal of Medical Physics/Association of Medical Physicists of India 38.1 (2013): 9.

[17] Viamonte, A. et al. Radiotherapy dosimetry using a commercial OSL system. Med. Phys. v. 35, n. 4, 2008.

[18] Dunn, $L$ et al. Commissioning of optically stimulated luminescence dosimeters for use in radiotherapy. Radiation Measurements. 51-52, p.31-39, 2013.

[19] Villani, D. et al. Application of optically stimulated luminescence 'nanoDot' dosimeters for dose verification of VMAT treatment planning using an anthropomorphic stereotactic end-to-end verification phantom. Radiation Measurements. 2017. http://dx.doi.org/10.1016/j.radmeas.2017.03.027

[20] Opp, D. et al. Validation of measurement-guided 3D VMAT dose reconstruction on a heterogeneous anthropomorphic phantom. J Appl Clin Med Phy. 14, 70-84, 2013.

[21] Perks, C.A., Le Roy, G., Prugnaud, B., 2007. Introduction of the InLight monitoring service. Radiat. Prot. Dosim. 125 (1-4), 220-223.

[22] Yahnke, C. J. Calibrating the microStar. 2009. Available in <http://www.landauer.com/uploadedFiles/Resource_Center/microStar\%20Calibration $\% 20$ and\%20Usage\%20Instructions\%2010-Jun-09.pdf>. 